\title{
Comunicação
}

[Communication]

\section{Efeito do tempo após a ingestão de leite sobre o equilíbrio ácido-básico de bezerros}

[Effect of the time after milk feeding on the acid-base balance of calves]

\author{
J.A.N. Lisbôa ${ }^{1}$, F.J. Benesi ${ }^{2}$, M.L.R. Leal' ${ }^{2}$, C.M.C. Teixeira ${ }^{2}$ \\ ${ }^{1}$ Departamento de Clínicas Veterinárias, Universidade Estadual de Londrina \\ Campus Universitário \\ 86051-990 - Londrina, PR \\ ${ }^{2}$ Departamento de Clínica Médica, Faculdade de Medicina Veterinária e Zootecnia, da USP, São Paulo, SP
}

Projeto financiado pela FAPESP $\left(\mathrm{n}^{\circ}\right.$ 98/01628-1)
Recebido para publicação em 5 de agosto de 2002
Recebido para publicção, após modificações, em 9 de julho de 2003
E-mail: janlisboa@uel.br

Informações sobre os possíveis efeitos da dieta sobre o equilíbrio ácido-básico de bezerros são escassas na literatura e os resultados mostram-se, por vezes, conflitantes. De forma geral, e com exceção das evidências apontadas por Reece e Hotchkiss (1987) e por Slanina et al. (1992), demonstrou-se que os animais lactentes tendem a se manter em estado de alcalose metabólica, ou a apresentar maior reserva alcalina em relação aos não lactentes (Reece, Wahlstrom, 1972; Reece, 1980, 1984; Thielscher, 1992). Tal fato poderia ser resultado da maior secreção de ácidos pelas células parietais da mucosa do abomaso que se intensifica no período pós-prandial (Reece, Wahlstrom, 1972). O objetivo deste trabalho foi investigar o efeito do tempo após a ingestão de leite sobre as variáveis representativas do equilíbrio ácidobásico em bezerros neonatos.

Oitenta bezerras sadias da raça Holandesa, entre 4 e 30 dias de idade, as quais integraram um estudo mais abrangente sobre o equilíbrio ácido-básico (Lisbôa, 2000), foram selecionadas para compor dois grupos de 40 animais cada. No primeiro grupo, colheu-se sangue imediatamente ou até, no máximo, 30 minutos após a refeição. No segundo, o sangue foi colhido com pouco mais de duas horas após a ingestão de leite. Respeitou-se a equivalência de idade das bezerras nos dois grupos e incluíram-se animais somente a partir do quarto dia de idade por já estarem adaptados ao sistema de oferta do leite em baldes, o que garantiu a ingestão de praticamente todo o volume de dois litros.

Amostras de sangue foram colhidas mediante punção da veia jugular e mantidas em condição de anaerobiose, empregando-se seringa plástica de $3 \mathrm{ml}$ com o seu espaço morto previamente preenchido com cerca de 400UI de heparina sódica (Liquemine; Roche; São Paulo.). As seringas foram mantidas submersas em banho de água gelada entre zero e $4^{\circ} \mathrm{C}$ (Lisbôa et al., 2001), por até quatro horas. Determinaram-se potencial hidrogeniônico $(\mathrm{pH})$, pressões parciais de dióxido de carbono $\left(\mathrm{pCO}_{2}\right)$ e de oxigênio $\left(\mathrm{pO}_{2}\right)$, bicarbonato $\left(\mathrm{HCO}_{3}{ }^{-}\right)$, dióxido de carbono total $\left(\mathrm{TCO}_{2}\right)$, excesso de bases $(\mathrm{BE})$, bicarbonato padrão $(\mathrm{StB})$ e saturação de oxigênio $\left(\mathrm{Sat}_{2}\right)$ utilizando-se um analisador automático de $\mathrm{pH}$ e de gases sangüíneos (Hemogasômetro ABL 5; Radiometer; Copenhaguem; Dinamarca.). Os resultados foram comparados por meio do teste t, admitindo-se a probabilidade de erro de $5 \%$.

As bezerras alimentadas há mais de duas horas exibiram valores médios significativamente mais altos para $\mathrm{pCO}_{2}, \mathrm{HCO}_{3}^{-}, \mathrm{TCO}_{2}, \mathrm{BE}$ e StB do que os animais com colheitas logo após a ingestão de leite (Tab. 
1). Estes resultados são coerentes com os de Reece e Wahlstrom (1972) e caracterizam o desenvolvimento de uma reserva alcalina maior poucas horas após o aleitamento.

Tabela 1. Valores médios ( $\overline{\mathrm{X}} \pm \mathrm{EP}$ ) das variáveis hemogasométricas observados no sangue venoso de bezerras da raça Holandesa, entre 4 e 30 dias de vida, de acordo com os tratamentos

\begin{tabular}{lcc}
\hline \multirow{2}{*}{ Variável } & \multicolumn{2}{c}{$\begin{array}{c}\text { Tempo entre o aleitamento e a } \\
\text { colheita do material }\end{array}$} \\
\cline { 2 - 3 } & até 30 minutos & acima de 2 horas \\
\hline $\mathrm{n}$ & 40 & 40 \\
Idade (dias) & $10,07 \pm 5,42$ & $10,15 \pm 5,24$ \\
$\mathrm{pH}$ & $7,360 \pm 0,027$ & $7,359 \pm 0,026$ \\
$\mathrm{pCO}_{2}(\mathrm{mmHg})^{* *}$ & $50,975 \pm 3,400$ & $54,980 \pm 3,516$ \\
$\mathrm{pO}_{2}(\mathrm{mmHg})$ & $29,175 \pm 5,952$ & $27,940 \pm 4,569$ \\
$\mathrm{HCO}_{3}(\mathrm{mmol} / \mathrm{l})^{* *}$ & $27,475 \pm 2,309$ & $29,468 \pm 2,265$ \\
$\mathrm{TCO}_{2}(\mathrm{mmol} / \mathrm{l})^{* *}$ & $28,900 \pm 2,426$ & $30,965 \pm 2,244$ \\
$\mathrm{BE}(\mathrm{mmol} / \mathrm{l})^{* *}$ & $2,500 \pm 2,339$ & $4,188 \pm 2,235$ \\
$\mathrm{StB}(\mathrm{mmol} / \mathrm{l})^{* *}$ & $25,800 \pm 2,115$ & $27,233 \pm 2,041$ \\
$\mathrm{Sat} \mathrm{O}_{2}(\mathrm{mmol} / \mathrm{l})$ & $45,100 \pm 13,301$ & $41,693 \pm 10,006$ \\
\hline$* * \mathrm{P}<0,01$ & \multicolumn{2}{l}{}
\end{tabular}

Do ponto de vista fisiológico, o processo de digestão do leite no abomaso está implicado com um rápido acúmulo de bases no compartimento fluido extracelular, em decorrência da secreção do ácido clorídrico ( $\mathrm{HCl})$ pelas células parietais glandulares (Kolb, 1984). Durante o processo, para cada $\mathrm{H}^{+}$secretado no lúmem abomasal, uma molécula de $\mathrm{HCO}_{3}{ }^{-}$é gerada e retorna à circulação. A secreção do $\mathrm{HCl}$ é de fundamental importância para a atividade das enzimas proteolíticas, renina e pepsina, as quais possuem o máximo poder catalisador sob condições de pH muito baixo, entre 2,0 e 3,5. Segundo Reece e Wahlstrom (1972), a secreção ácida do abomaso pode se continuar por até seis horas após a ingestão de leite, quando o pH ideal é alcançado.

$\mathrm{O}$ aumento simultâneo da $\mathrm{pCO}_{2}$ poderia ser interpretado como uma resposta compensatória do organismo frente a essa condição transitória de bases acumuladas. A hipoventilação pulmonar causaria uma certa retenção de $\mathrm{CO}_{2}$ evitando-se a elevação do pH sangüíneo (Houpt, 1984).

Segundo Reece e Wahlstrom (1972), Reece (1980, 1984) e Thielscher (1992), a alcalose metabólica com compensação respiratória parcial é o estado ácido-básico naturalmente mantido no bezerro lactente, tendendo a se intensificar no período pós-prandial. Os resultados não confirmam essa afirmação, pois até mesmo o aumento da reserva alcalina observado horas depois da ingestão do leite não pode caracterizar a presença de qualquer grau de desequilíbrio aparente. Os autores mencionados tomaram como base para o julgamento de suas interpretações os valores limítrofes de 7,4 para o pH, de 40,0 mmHg para a pCO ${ }_{2}$, de 24,0 mmol/l para o $\mathrm{HCO}_{3}{ }^{-}$e de até 4,0 mmol/l para o BE, os quais são considerados fisiológicos para a espécie humana, mas não devem ser aceitos para os bovinos neonatos (Lisbôa, 2000).

Parece mais coerente admitir que, em decorrência do tipo de dieta e dos aspectos fisiológicos apontados anteriormente, os bezerros, enquanto lactentes, podem manter-se com uma reserva alcalina mais elevada. O momento da colheita da amostra após a ingestão de leite deve ser padronizado em estudos sobre o equilíbrio ácido-básico e considerado na interpretação de resultados hemogasométricos.

Palavras-chave: bezerro lactente, equilíbrio ácido-básico

\section{ABSTRACT}


Efeito do tempo após a ingestão de leite...

Blood pH, carbon dioxide and oxygen partial pressures, bicarbonate, total carbon dioxide, base excess, standard bicarbonate and oxygen saturation concentrations were measured by blood gas analyses in a study of assessment of the acid-base status in young calves. Venous blood samples were taken from 80 healthy female Holstein calves, from four to 30 days of age. The calves were divided in two groups based on the interval between the morning milk feeding and the blood sample collection (up to 30 minutes or over two hours). Greater alkali reserve and higher carbon dioxide pressure values were observed later than $2 \mathrm{~h}$ after milk feeding, supporting the influence of the diet on the acid-base balance of calves during the milk feeding phase.

Keywords: nursing calf, acid-base balance

\section{REFERÊNCIAS BIBLIOGRÁFICAS}

HOUPT, R.T. Water, electrolytes, and acid-base balance. In: SWENSON, M.J. Duke's physiology of domestic animals. 10.ed. Ithaca: Cornell University Press, 1984. p.486-506.

KOLB, E. Fisiologia veterinária. 4.ed. Rio de Janeiro: Guanabara Koogan, 1984. 621p.

LISBÔA, J.A.N. Equilíbrio ácido-básico em bezerras sadias da raça holandesa, no primeiro mês de vida. Influência do fator etário. 2000. 113p. Tese (Doutorado em Clínica Veterinária) - Faculdade de Medicina Veterinária e Zootecnia, Universidade de São Paulo, São Paulo.

LISBÔA, J.A.N.; BENESI, F.J.; MARUTA, C.A. et al. Tempo de viabilidade de amostras de sangue venoso bovino destinadas ao exame hemogasométrico, quando mantidas sob conservação em água gelada. Ciên. Rural, v.31, p.271-276, 2001.

REECE, W.O. Acid-base balance and selected hematologic, electrolyte, and blood chemical variables in calves: milk-fed vs conventionally fed. Am. J. Vet. Res., v.41, p.109-113, 1980.

REECE, W.O. Acid-base balance and selected hematologic, electrolytic, and blood chemical variables in calves nursing cows: one week through fifteen weeks. Am. J. Vet. Res., v.45, p.666-669, 1984.

REECE, W.O.; HOTCHKISS, D.K. Blood studies and performance among calves reared by different methods. J. Dairy Sci., v.70, p.1601-1611, 1987.

REECE, W.O.; WAHLSTROM, J.D. Variations in plasma composition of calves: Relationship of acidbase status to calf age, ration, and feeding time. Am. J. Vet. Res., v.33, p.2169-2174, 1972.

SLANINA, L.; NAGY, O.; SEDOVIC, M. et al. Dynamik des Säure-Basen-Haushaltes des venösen und arteriellen Blutes bei klinisch gesunden Kälbern. Dtsch. Tierärztl. Wschr., v.99, p.182-186, 1992.

THIELSCHER, H.H. Der Säure/Basen-Status im Blut von Kälbern bei Mutterkuhhaltung und in der Milchmast. J. Vet. Med. A, v.39, p.531-540, 1992. 